\section{Commentary: Enhancing risk assessment by incorporating more of what we know}

Eugene H. Blackstone, MD, ${ }^{\mathrm{a}, \mathrm{b}}$ Amol Pande, $\mathrm{PhD},{ }^{\mathrm{a}}$ and Jeevanantham Rajeswaran, $\mathrm{PhD}^{\mathrm{b}}$

(f) Supplemental material is available online.

The closest preoperative values of variables to time of surgery almost exclusively populate risk assessment models, with the notable exception of time since most recent acute myocardial infarction. ${ }^{1}$ In their article in this issue of the Journal, Griffin and colleagues ${ }^{2}$ remind us that the degree of organ function deterioration may be a more powerful risk factor than traditional preoperative values. In this commentary, we consider 6 features of preoperative variables that can be incorporated into multivariable models and provide computer code (Appendix E1) and mathematical (Appendix E2) descriptions.

\section{FEATURE 1: SINGLE VALUES REFLECTING PREOPERATIVE PATIENT/ORGAN STATUS}

These "baseline" values are implicitly assumed to be static and, if continuous, measured without error. They are incorporated straightforwardly as covariates in multivariable models.

\section{FEATURE 2: AVERAGE OF MULTIPLE ( $>$ 1) MEASUREMENTS OF SAME PREOPERATIVE VARIABLE}

This is typical of echocardiographic measurements in which values from 3 or more heartbeats are averaged.

From the a Department of Thoracic and Cardiovascular Surgery, Heart, Vascular, and Thoracic Institute and ${ }^{\mathrm{b}}$ Department of Quantitative Health Sciences, Lerner Research Institute, Cleveland Clinic, Cleveland, Ohio.

Disclosures: The authors reported no conflicts of interest.

The Journal policy requires editors and reviewers to disclose conflicts of interest and to decline handling or reviewing manuscripts for which they may have a conflict of interest. The editors and reviewers of this article have no conflicts of interest.

Received for publication March 30, 2020; revisions received March 30, 2020; accepted for publication March 31, 2020; available ahead of print April 12, 2020.

Address for reprints: Eugene H. Blackstone, MD, Clinical Investigations, Heart, Vascular, and Thoracic Institute, Cleveland Clinic, Desk JJ40, 9500 Euclid Ave, Cleveland, OH 44195 (E-mail: blackse@ccf.org).

J Thorac Cardiovasc Surg 2022;163:1388-90 $0022-5223 / \$ 36.00$

Copyright $₫ 2020$ Published by Elsevier Inc. on behalf of The American Association for Thoracic Surgery

http://dx.doi.org/10.1016/j.jtcvs.2020.03.132

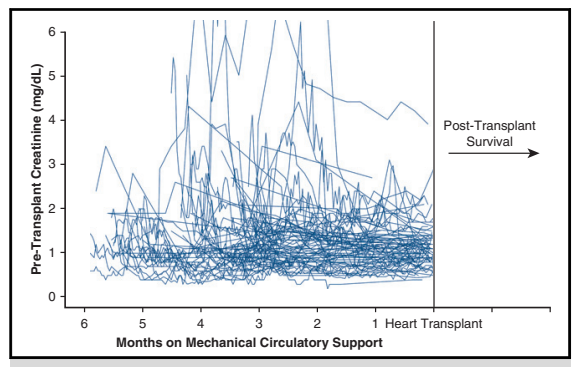

Individual creatinine trajectories for discovery of effect on post-transplantation survival.

CENTRAL MESSAGE

Although preoperative baseline values predominate risk assessment models, other features of a patient's state, such as rapidity of renal function deterioration, may be even more informative.

The average is the covariate entered into the multivariable models. Information lost by this is beat-to-beat variability.

\section{FEATURE 3: AREA UNDER A "CURVE" OF MULTIPLE VALUES}

This is a refined version of feature 2 , in that a variable such as atrial fibrillation is observed multiple times across time (as might become commonplace with wearables) to provide information about disease burden over a designated period. ${ }^{3}$ This feature can be estimated directly from the longitudinal sequence of preoperative measures or from a fitted temporal trend (smoothed version of raw data ${ }^{4}$ ) using integration.

\section{FEATURE 4: VALUES BASED ON CLINICAL CRITERIA}

This is what Griffin and colleagues have presented, in which 2 values were measured, the first being the lowest creatinine value measured in the 3 months before surgery and the second being the measurement immediately before surgery. ${ }^{2}$ They entered the difference between these 2 measurements into their multivariable model. (Note that use of "change scores" like this has important statistical drawbacks. ${ }^{5,6}$ ) Because measurements cannot be made continuously, it is unknown what the lowest creatinine value was in that 3-month interval. 


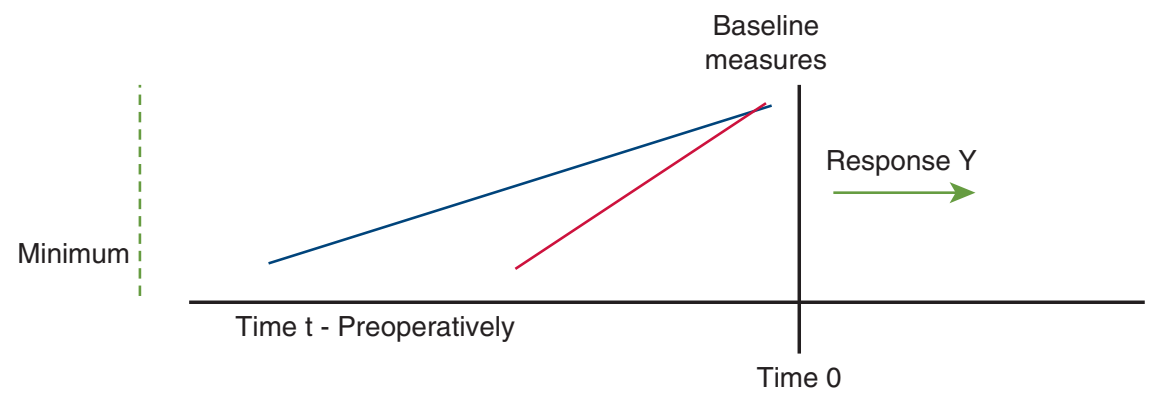

FIGURE 1. Importance of recording time of preoperative measurements. Illustrated are values for some preoperative variables, such as creatinine, for 2 patients (blue and red lines) who have the same minimum value and an immediate preoperative measurement. Considering just the difference between these 2 measurements as in Griffin and colleagues ${ }^{2}$ produces the same value (see feature 2). However, 1 patient had a more gradual increase over a longer time (lower slope), as shown in blue, than the other patient with the same increase, but over a shorter time interval (steeper slope). These different slopes may have different effects on the outcome (Response Y), despite the identical immediate preoperative value.

\section{FEATURE 5: INDIVIDUAL PATIENT SLOPES OF VALUES}

This feature is what is missing from the article: a temporal slope of preoperative creatinine. Had timing of the measurements been known, an approximation of the slope could have been estimated and used in their analysis (Figure 1).

\section{FEATURE 6: TEMPORAL PATIENT-SPECIFIC TRAJECTORY OF VALUES}

Feature 6 is more advanced than feature 5 , incorporating all observed longitudinal measurements $(>2)$, or smoothed version thereof, and time of measurement (Figure 2) directly into the multivariable outcomes model, a timevarying coefficient model. ${ }^{7}$ Smoothed values can be obtained by linear or nonlinear mixed-effects parametric models $^{8}$ or by a nonparametric approach. ${ }^{4}$

\section{CONCLUSIONS}

All these features can be implemented in 2 steps using readily available software. Step 1 is estimating the feature, and step 2 is using the feature as a covariate in multivariable models of outcomes. For features 5 and 6 , these 2 steps can be accomplished simultaneously by joint modeling.'

Incorporating such features could, as Griffin and colleagues state, enhance risk models and "trigger heightened vigilance in the postoperative period." $"$

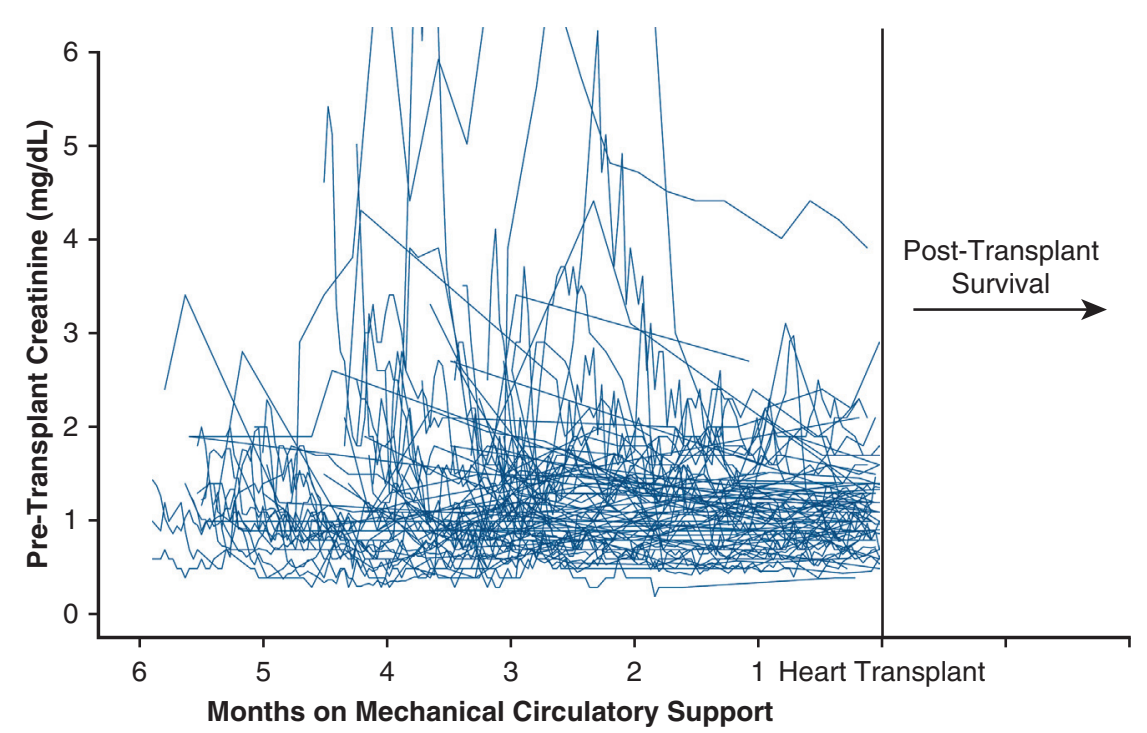

FIGURE 2. Effect of repeated measurements of pretransplantation renal function on survival after heart transplantation. Each line depicts an observed patient-specific longitudinal profile of pretransplantation creatinine over the 6 months before transplantation. 


\section{References}

1. O’Brien SM, Feng L, He X, Xian Y, Jacobs JP, Badhwar V, et al. The Society of Thoracic Surgeons 2018 adult cardiac surgery risk models, part 2: statistical methods and results. Ann Thorac Surg. 2018;105:1419-28.

2. Griffin BR, Bronsert M, Reece TB, Pal JD, Cleveland JC, Fullerton DA, et al. Creatinine elevations from baseline at the time of cardiac surgery are associated with postoperative complications. J Thorac Cardiovasc Surg. 2022;163:1378-87.

3. Blackstone EH, Chang HL, Rajeswaran J, Parides MK, Ishwaran H, Li L, et al; Cardiothoracic Surgical Trials Network Investigators. Biatrial maze procedure versus pulmonary vein isolation for atrial fibrillation during mitral valve surgery: new analytical approaches and end points. J Thorac Cardiovasc Surg. 2019;157: 234-43.e9.

4. Hurst TE, Xanthopoulos A, Ehrlinger J, Rajeswaran J, Pande A, Thuita L, et al. Dynamic prediction of left ventricular assist device pump throm- bosis based on lactate dehydrogenase trends. ESC Heart Fail. 2019;6: 1005-14.

5. Vickers AJ, Altman DG. Statistics notes: analysing controlled trials with baseline and follow up measurements. BMJ. 2001;323:1123-4.

6. Vickers AJ. The use of percentage change from baseline as an outcome in a controlled trial is statistically inefficient: a simulation study. BMC Med Res Methodol. 2001;1:6.

7. Fan J, Zhang W. Statistical methods with varying coefficient models. Stat Interf. 2008;1:179-95

8. Rajeswaran J, Blackstone EH. A multiphase non-linear mixed effects model: an application to spirometry after lung transplantation. Stat Methods Med Res. 2017;26:21-42.

9. Rajeswaran J, Blackstone EH, Barnard J. Joint modeling of multivariate longitudinal data and competing risks using multiphase submodels. Stat Biosci. 2018;10:651-85.

\section{Commentary: Absolute creatinine values and preoperative acute kidney injury in cardiac surgery patients: Are we barking up the wrong tree?}

\author{
Carly Lodewyks, MD, MSc, ${ }^{\mathrm{a}, \mathrm{b}}$ and \\ Rakesh C. Arora, MD, $\mathrm{PhD}^{\mathrm{a}, \mathrm{b}}$
}

Acute kidney injury (AKI) has been shown to occur in up to $22.3 \%$ of patients following cardiac surgery. ${ }^{1}$ Multiple studies have demonstrated that pre- and post-operative AKI is associated with poor postoperative outcomes. ${ }^{1-3}$ Previously, Hobson and colleagues ${ }^{2}$ demonstrated that postoperative AKI was an independent predictor of mortality (hazard ratio, 1.39, 95\% confidence interval [CI], 1.23-1.57). In a large database study, Cooper and colleagues $^{3}$ found that declining preoperative estimated glomerular filtration rate was associated with increased operative mortality and increased postoperative AKI

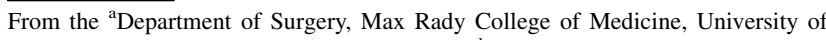
Manitoba, Winnepeg, Manitoba, Canada; and ${ }^{\mathrm{b}}$ Cardiac Sciences Program, Section of Cardiac Surgery, St Boniface Hospital, Winnipeg, Manitoba, Canada.

Disclosures: Dr Arora has received an unrestricted educational grant from Pfizer Canada, Inc, and honoraria from Mallinckrodt Pharmaceuticals, Abbott Nutrition, and Edwards Lifesciences for work unrelated to this work. The other author has nothing to disclose with regard to commercial support.

Received for publication March 21, 2020; revisions received March 21, 2020; accepted for publication March 21, 2020; available ahead of print April 9, 2020.

Address for reprints: Rakesh C. Arora, MD, PhD, Cardiac Sciences Program, Section of Cardiac Surgery, St Boniface Hospital, CR3012-369 Tache Ave, Winnipeg,

Manitoba R2H2A6, Canada (E-mail: rakeshcarora@gmail.com).

J Thorac Cardiovasc Surg 2022;163:1390-1

$0022-5223 / \$ 36.00$

Copyright (c) 2020 by The American Association for Thoracic Surgery

http://dx.doi.org/10.1016/j.jtcvs.2020.03.093

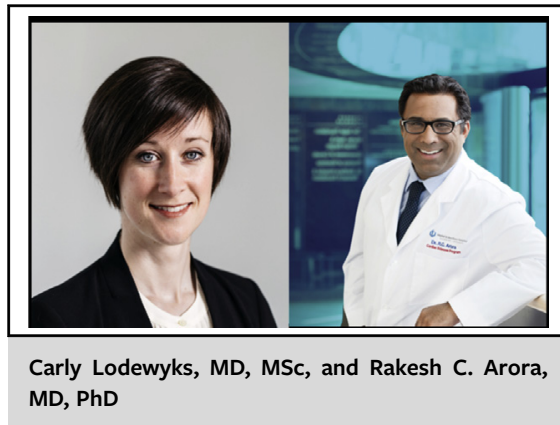

CENTRAL MESSAGE

AKI is an important cause of morbidity and mortality after cardiac surgery. Small changes in preoperative creatinine from baseline may also be harmful; however, the accuracy of this needs to be confirmed in future studies.

requiring dialysis, stroke, infection, prolonged ventilation, and hospital length of stay.

Until recently, the effect of preoperative kidney disease on postoperative outcomes has been investigated using an absolute creatinine value or single calculated estimated glomerular filtration rate. Griffin and colleagues ${ }^{4}$ sought to determine whether an acute change in preoperative creatinine from a baseline value would significantly influence postoperative outcomes in cardiac surgery patients. In this retrospective study of 1486 patients undergoing nonemergency cardiac surgery, a creatinine change from baseline (minimum creatinine available 3 months after surgery) to the presurgical value was calculated. They observed an 


\section{APPENDIX E1. SAS CODE FOR FEATURE 5 \\ Jeevanantham Rajeswaran, PhD}

\section{Two-Step Approach}

Step 1: Linear mixed-effects model for longitudinal preoperative measures

Step 2: Cox proportional hazards model for time to event, where patient-specific slopes, which are estimated using a linear mixed-effects model in step 1, are entered as a covariate in the time-to-event model.

Dataset - Built: Multiple record dataset

/**********Variables $* * * * * * * * * *$ /

ptid - patient ID

iv_test - time to a preoperative blood testing

creat_pr - preoperative longitudinal measurement of creatinine level

event - (indicator) time to event after time zero (time of surgery)

iv_event - time to the event or censoring

$\mathrm{X}$ - other baseline covariates

/ $* * * * * * * * * * * * * * * * * * * * * * * * * * * * /$

/*******Step 1: Linear Mixed-Effects Model ******/

proc sort data=built; by ptid iv_test; run;

ods trace on;

ods output parameterestimates=est.peML_avmngr2pt_ab; proc nlmixed data $=$ built $\mathrm{gconv}=1 \mathrm{E}-8$ qpoints $=1$;

parms $/ * * *$ Starting values $* /$

/* overall intercept and slope*/

$\mathrm{a} 0=-0.1346 \mathrm{~b} 1=0.0721$

/* Normal model variance for the response */ logsig $=-0.325$

/* Random effects variance (for intercept and slope)*/

$\log \operatorname{sigu}=-0.5822 \log \operatorname{sigv}=-1.7407 \operatorname{suv}=0.02201$

;

1_eta $=(\mathrm{a} 0+\mathrm{u})+(\mathrm{b} 1+\mathrm{v}) * \mathrm{iv} \_$test;

model creat_pr $\sim \operatorname{normal}\left(1 \_\right.$eta, $\left.\exp (2 * \log \operatorname{sig})\right)$;

random u v $\sim \operatorname{normal}([0,0],[\exp (2 * \log \operatorname{sigu})$, suv, $\exp (2 * \log \operatorname{sigv})])$ subject $=$ ptid out=rand_out;

run; ods trace off;

/* Extract estimated patient-specific slopes */

data randbi; set rand_out; where Effect='v';

keep ptid estimate b1;

rename estimate $=\mathrm{b} 1 \mathrm{i}$;

data randbi; set randbi; beta_i $=(\mathrm{b} 1 \mathrm{i}+\mathrm{b} 1)$; run;

/**Merge with original data */

proc sql;

create table built_b as select $*$ from built

as left join randbi

quit;
/****Step 2: Cox PH regression - Response model $* * * /$

Proc phreg data=built_bi; model iv_event $*$ event $=$ beta_i $\times 1 \times 2 \times 3 / *$ can include other covariates $* /$

Run;

\section{Joint Model Approach}

This code is for joint modeling using a linear mixedeffects model for the longitudinal preoperative measures and a Cox-type proportional hazards model for the time to event. Patient-specific slopes are estimated using the linear mixed-effects model and used as a covariate in the time-toevent model. Note that this code fits the 2 models simultaneously. If each patient has sufficient preoperative longitudinal data, the models can be fitted in 2 steps as described above.

Dataset - Built: Multiple record dataset

/***********Variables $* * * * * * * * * *$ /

ptid - patient ID

iv_test - time to a preoperative blood testing

creat_pr - preoperative longitudinal measurement of creatinine level

event - (indicator) time to event after time zero (time of surgery)

iv_event - time to the event or censoring

X - other baseline covariates

/****************************/

proc sort data=built; by ptid iv_test; run;

proc nlmixed data $=$ built gconv $=1 \mathrm{E}-8$ qpoints $=1$;

parms /* Starting values*/

/***************************************/

/**Longitudinal Data - overall intercept and slope $* *$ / $\mathrm{a} 0=-0.1353 \mathrm{~b} 1=0.072$

/* Normal model variance -normality assumption of preoperative measure*/

$\log \operatorname{sig}=-0.324$

/* Random effects variance (random intercept and random slope)*/

$\log \operatorname{sig} \mathrm{u}=-0.5806 \log \operatorname{sigv}=-1.7395 \mathrm{suv}=0.02139$

/***************************************/

/* Time to Event : data $* * * * * /$

/* Late Increasing hazard*/

thalf $11=3.2264$ nu $1=-0.2997$

/* covariate in the time to event model $*$ /

$\mathrm{s} 1=0.111=-1.287212=0.01760$

;

| $* * * * * * * * * * * * * * * * * * * * * * * * * * * * * * * * * * * * * * * * * * * * /$

|**********Longitudinal data: Likelihood $* * * * * * * *$ |

/************************************************/

/* Linear mixed effects model $*$ /

1_eta $=(\mathrm{a} 0+\mathrm{u})+(\mathrm{b} 1+\mathrm{v}) *$ iv_test;

resid $=$ (creat_pr - 1_eta);

sigmasq $=\exp (2 * \log s i g)$; 


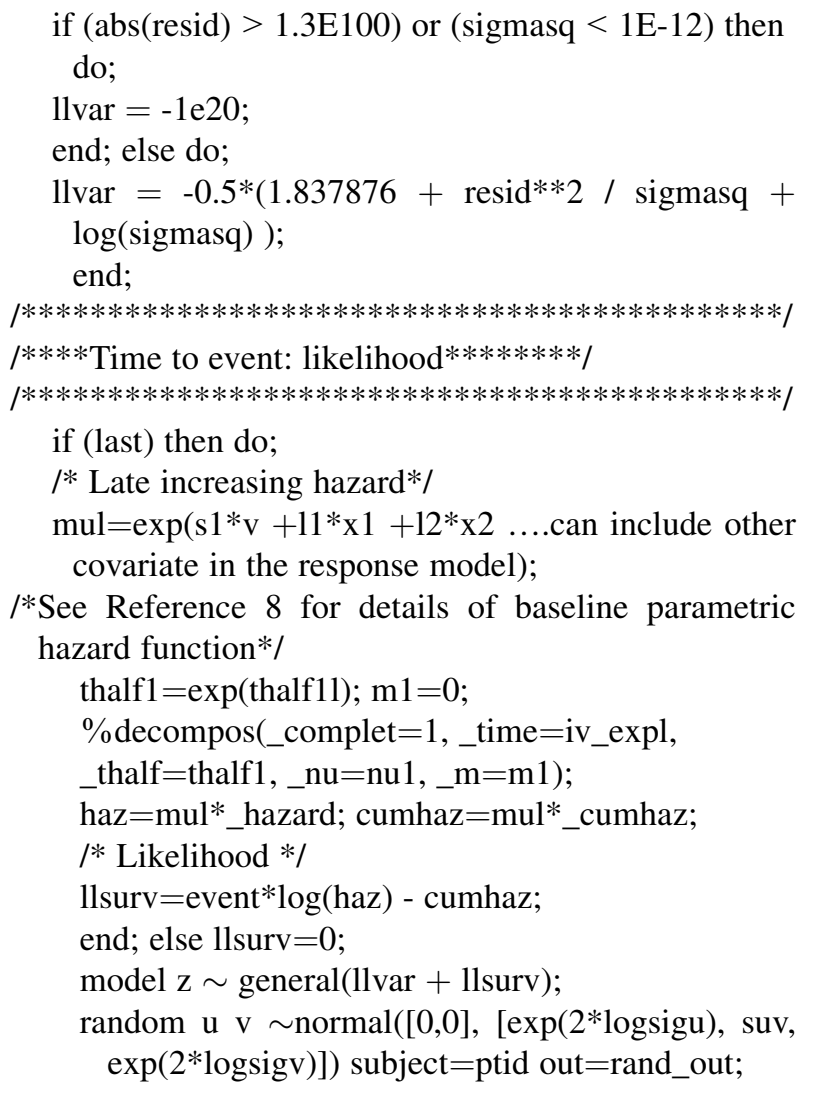

run;

ods trace off;

\section{APPENDIX E2: APPENDIX E2. MATHEMATICAL DISCUSSION}

Amol Pande, PhD

This Appendix presents various strategies for incorporating preoperative information to assess its association with postoperative events using formal mathematical notation.

\section{Setup}

For the $i$ th patient, where $i=1,2, \ldots, \mathrm{n}$, let $\mathrm{T}_{i}$ represent event or censored time, and let $x_{i}^{(k)}$, for $\mathrm{k}=1,2, \ldots, \mathrm{P}$, represent preoperative information that does not vary with time.

A preoperative variable that stands out in the work of Griffin and colleagues ${ }^{\mathrm{E} 1}$ is preoperative creatinine. We use $z_{i}$, for $i=1,2, \ldots, \mathrm{n}$, to denote this variable. In the study of Griffin and colleagues, each patient had at least 2 creatinine measurements of $z_{i}$, and the authors took the difference between the lowest and immediate preoperative values. For the purpose of this Appendix, we assume that there could be a single measurement or multiple measurements of the preoperative variable per patient, that is, $z_{i}=\left(z_{i, 1}, z_{i}, 2, \ldots, z_{i}, n_{i}\right)$, and although not measured by Griffin and colleagues, we assume that for multiple measurements of $z_{i}$ per patient, there is an accompanying variable denoted by $u_{i}$, for $i=1,2, \ldots, n$. For example, $u_{i}$ could be the time at which the measurement was made or an increasing index at which $z_{i}$ is collected.

\section{Model}

For the purpose of this Appendix, we use the Cox proportional hazards model for the outcome as a time-related event. This model assumes that the hazard function has the following form:

$$
\mathrm{h}\left(\mathrm{t} \mid x_{i}^{(1)}, \ldots, x_{i}^{(\mathrm{P})}, z_{i} u_{i}\right)=\mathrm{h}_{0}(\mathrm{t}) \exp \left(\mathrm{f}\left(x_{i}^{(1)}, \ldots, x_{\mathrm{i}}^{(\mathrm{P})}, z_{i} u_{i}\right)\right),
$$

for $i=1,2, \ldots, n$,

where $h_{0}(t)$ is a baseline hazard function, and $f()$ is either an unknown function estimated from the data or, in most cases, the user-specified linear function. Note that if the information for $u_{i}$ is not available, it can be suppressed in this model.

\section{Strategies for Incorporating Preoperative Information}

Here we provide strategies for incorporating $z_{i}$ (and $u_{i}$, if available) in the above model. We assume a linear functional form for $\mathrm{f}(\mathrm{)})$, although it can be generalized to account for nonlinearities, and even interactions, among variables.

Feature 1: Each patient has a single measurement of $z_{i}$, and the measurement of $u_{i}$ is not available. The linear functional form of $f()$ can be written as

$$
f\left(x_{i}^{(1)}, \ldots, x_{i}^{(\mathrm{P})}, z_{i}\right)=\sum_{k=1}^{P} x_{i}^{(\mathrm{k})} \mathrm{b}_{\mathrm{k}}+z_{i} \mathrm{~b},
$$

where $b_{1}, \ldots, b_{\mathrm{P}}$, and $\mathrm{b}$ are unknown parameters that are estimated from the data. The Cox parameter estimation procedure has been described previously. ${ }^{\mathrm{E} 2}$

Feature 2: Patients have multiple measurements of $z_{i}$ but no measurements of $u_{i}$. Multiple measurements of $z_{i}$ are averaged, denoted by $\bar{z}_{i}$, for $i=1,2, \ldots, n$. The estimate of $\bar{z}_{i}$ will be more stable (ie, lower variance) than using any one of the measurements $z_{i, 1}, z_{i, 2}, \ldots, z_{i}, n_{i}$. The functional form of $f()$ can be written as

$$
f\left(x_{i}^{(1)}, \ldots, x_{i}^{(\mathrm{P})}, \bar{z}_{i}\right)=\sum_{k=1}^{P} x_{i}^{(\mathrm{k})} \mathrm{b}_{\mathrm{k}}+\bar{z}_{i} \mathrm{~b} .
$$

Feature 4: Each patient has at least 2 measurements of $z_{i}$, but the measurements of $u_{i}$ are not available. Instead of average, the difference between the lowest value and the immediate preoperative value is used-a strategy used by Griffin and colleagues. ${ }^{\mathrm{E} 1}$ Thus, we use $z_{i}^{*}=z_{i, i m}-\min \left(z_{i, 1}, z_{i, 2}, \ldots, z_{i}, n_{i}\right)$ for $i=1,2, \ldots, n$, where $z_{i, \mathrm{im}}$ is the immediate preoperative value for the $i$ th patient. The functional form of $\mathrm{f}(\mathrm{)}$ can be written as

$$
f\left(x_{i}^{(1)}, \ldots, x_{i}^{(\mathrm{P})}, z_{i}^{*}\right)=\sum_{k=1}^{P} x_{i}^{(\mathrm{k})} \mathrm{b}_{\mathrm{k}}+z_{i}^{*} \mathrm{~b} .
$$

Parameter $\mathrm{b}$ provides an association of the preoperative creatinine difference with posttransplantation survival. 
Owing to a lack of time measurements, parameter $b$ fails to identify patients with similar preoperative creatinine differences that are measured at different times. We address this in features 5 and 6.

Feature 5: A patient can have a single measurement or multiple measurements of $z_{i}$, and the corresponding measurements of $u_{i}$ are available. We incorporate $n_{i}$ measurements of $z_{i}$ and $u_{i}$, where $n_{i} \geq 1$, using the following linear random-effects model to extract the random slope for each patient:

$$
z_{i}=\alpha_{i}+\beta_{i} u_{i}+e_{i}, \text { for } i=1,2, \ldots, n,
$$

where $\alpha_{\mathrm{i}}$ and $\beta_{\mathrm{i}}$, respectively, represent the random intercept and the random slope. This model provides an estimate of $\beta_{\mathrm{i}}$ for each patient, which we use in $\mathrm{f}(\mathrm{)})$ as follows:

$$
f\left(x_{i}^{(1)}, \ldots, x_{i}^{(\mathrm{P})}, \beta_{i}\right)=\sum_{k=1}^{P} x_{i}^{(\mathrm{k})} \mathrm{b}_{\mathrm{k}}+\beta_{i} \mathrm{~b}_{\mathrm{slp}},
$$

where $b_{\text {slp }}$ is the regression parameter for the random slope. The random slope represents the patient-specific rate at which preoperative creatinine changes with time. The 2 models described here can be fitted using a 2-step approach or the slightly more complicated joint model approach described in Appendix E1.

Feature 6: The models considered in the preceding text are limited in terms of capability of capturing any nonlinear relationship of association. A nonlinear relationship can be obtained by considering the following model. To incorporate $\mathrm{n}_{\mathrm{i}}$ measurements of $z_{i}$ and $u_{i}$, where $n_{i} \geq 1$, we need to modify the dimensions of $x_{i}^{(k)}$, for $k=1, \ldots, \mathrm{P}$, so that all variables have the same dimensions; this should be reflected in the dimension of $\mathrm{f}(\mathrm{)})$. To do this, we replicate values of $x_{i}^{(k)} n_{i}$ times and form a vector $\mathrm{X}_{i}^{(k)}$ of $n_{i}$ dimension. The functional form of $\mathrm{f}()$, now in $n_{i}$ dimension, can be written as

$$
f\left(\tilde{X}_{i}^{(1)}, \ldots, \tilde{X}_{i}^{(\mathrm{P})}, \tilde{Z}_{i}, u_{i}\right)=\sum_{k=1}^{P} \tilde{X}_{i}^{(\mathrm{k})} \mathrm{b}_{\mathrm{k}} \mathbf{1}+\tilde{Z}_{i} \mathrm{~b}\left(u_{i}\right),
$$

where $\tilde{X}_{i}^{(k)}$ and $\tilde{Z}_{i}$ are diagonal matrix forms of $\mathrm{X}_{i}^{(k)}$ and $z_{i}$, respectively, for $k=1,2, \ldots, \mathrm{P}, \mathbf{1}$ is a vector of $n_{i}$ dimension, and $\mathrm{b}\left(u_{i}\right)$ is a function of $u_{i}$, which is different from parameter $b$ that we considered earlier. This model is referred to as a varying coefficient model. ${ }^{\mathrm{E} 3}$ Once the function $\mathrm{b}\left(u_{i}\right)$ is estimated, the plot of $u_{i}$ versus $\mathrm{b}\left(u_{i}\right)$ can illustrate possibly hidden trends not revealed in the linear model.

\section{E-References}

E1. Griffin BR, Bronsert M, Reece TB, Pal JD, Cleveland JC, Fullerton DA, et al Creatinine elevations from baseline at the time of cardiac surgery are associated with postoperative complications. J Thorac Cardiovasc Surg. 2020 [In press]

E2. Cox DR. Regression models and life-tables. J R Stat Soc Ser B Stat Methodol. 1972;34:187-220

E3. Fan J, ZhangW. Statistical methods with varying coefficient models. Stat Interf 2008:1:179-95 